\title{
Automedicação em idosos atendidos em uma unidade básica de saúde do município de Belém - Pará
}

\author{
Self-medication in the old-aged care in a basic health unit in the municipality of Belém - \\ Pará
}
Automedicación en atención a paciente mayor en una Unidad de Salud Básica en el municipio de Belém - Pará

Amanda Gabryelle Nunes Cardoso Mello ${ }^{1 *}$, Nathália Dias da Gama, Rosivana Borges da Silva ${ }^{1}$, Luann Wendel Pereira de Sena², Rozana Lúcia Pinheiro Castro ${ }^{3}$.

\section{RESUMO}

Objetivo: Analisar as causas da automedicação na pessoa idosa. Métodos: Trata-se de um estudo prospectivo descritivo, quantitativo, por aplicação de questionários aos idosos atendidos em uma UMS de Belém, Pará, o qual foi aprovado por Comitê de Ética em Pesquisa. Os dados foram analisados pelo teste qui-quadrado (x2), utilizando o programa Biostat 5.3. Resultados: A amostra apresentou maioria feminina $(62,5 \%)$, de 60 a 80 anos $(94,6 \%)$, ensino fundamental $(61,6 \%)$, casados $(36,6 \%)$ e aposentados ou pensionistas $(71,4 \%)$. As mulheres fazem mais uso de medicação $(p=0,0287)$ e leem a bula $(p=0,0013)$, sendo essa atitude atrelada ao elevado grau de escolaridade $(p=0,0078)$. Entretanto, a automedicação diminui com o aumento da idade $(p=0,0245)$ e o uso de plantas medicinais e fitoterápicos está atrelado de 60 a 80 anos $(p=0,0208)$ e àqueles com baixa ou sem renda comprovada $(p=0,0103)$. A baixa escolaridade leva 0 desconhecimento do tempo de manifestação dos sintomas iniciais, induzindo o uso indiscriminados de medicamentos $(p=0,0067)$ e influenciando na diminuição $(p=0,0022)$ da procura por atendimento médico ou profissional de saúde para correta orientação. Conclusão: Conclui-se que os idosos necessitam de mais orientação correta ao uso de medicamentos e plantas medicinais, com maior participação dos profissionais de saúde.

Palavras-chave: Idoso, Automedicação, Profissionais de saúde.

\begin{abstract}
Objective: To analyze the causes of self-medication in the old-aged patient. Methods: This is a prospective, descriptive, quantitative study, by applying questionnaires to the old-aged patient attended at a UMS in Belém, Pará, which was approved by the Research Ethics Committee. The data were analyzed using the chi-square test (x2), using the Biostat 5.3 program. Results: The sample had a female majority (62.5\%), 60 to 80 years old $(94.6 \%)$, elementary school $(61.6 \%)$, married $(36.6 \%)$ and retirees or pensioners $(71.4 \%)$. Women make more use of medication ( $p=0.0287)$ and read the package leaflet $(p=0.0013)$, this attitude being linked to the high level of education $(p=0.0078)$. However, self-medication decreases with increasing age $(p=0.0245)$ and the use of medicinal plants and herbal medicines is linked to 60 to 80 years old $(p=0.0208)$ and to those with low or no proven income $(p=0.0103)$. Low education leads to ignorance of the time of manifestation of initial symptoms, inducing the indiscriminate use of medications $(p=0.0067)$ and influencing the decrease $(p=0.0022)$ in the search for medical attention or health professionals for correct guidance. Conclusion: The old-aged patient need more correct guidance on the use of medicines and medicinal plants, with a greater participation of health professionals.
\end{abstract}

Keywords: Elderly, Self-medication, Health professionals.

\footnotetext{
${ }^{1}$ Faculdade Pan Amazônica (FAPAN), Belém - PA. *E-mail: amandagncmello@yahoo.com.br

${ }^{2}$ Escola Superior da Amazônia (ESAMAZ), Belém - PA.

${ }^{3}$ Secretária Municipal de Saúde de Belém (SESMA), Belém - PA.
}

SUBMETIDO EM: 6/2020

ACEITO EM: 7/2020

PUBLICADO EM: 11/2020 


\section{RESUMEN}

Objetivo: Analizar las causas de la automedicación en los ancianos. Métodos: Este es un estudio prospectivo, descriptivo, cuantitativo, mediante la aplicación de cuestionarios a los ancianos atendidos en un UMS en Belém, Pará, que fue aprobado por el Comité de Ética en Investigación. Los datos se analizaron utilizando la prueba de chi-cuadrado, utilizando el programa Biostat 5.3. Resultados: La muestra tenía una mayoría femenina (62.5\%), 60 a 80 años $(94.6 \%)$, escuela primaria $(61.6 \%)$, casada (36.6\%) y jubilados o pensionados $(71.4 \%)$ Las mujeres usan más los medicamentos $(\mathrm{p}=0.0287)$ y leen el prospecto $(\mathrm{p}=0.0013)$, que está relacionado con un alto nivel de educación $(p=0.0078)$. Las plantas medicinales y las hierbas medicinales están vinculadas entre 60 y 80 años ( $p=0.0208)$ y aquellas con ingresos bajos o nulos $(p=0.0103)$. La baja educación conduce a la ignorancia del tiempo de manifestación de los síntomas iniciales, lo que induce el uso indiscriminado de medicamentos $(p=0.0067)$ e influye en la disminución ( $p=0.0022)$ en la búsqueda de atención médica o profesionales de la salud para obtener una orientación correcta. Conclusión: Los ancianos necesitan una orientación más correcta sobre el uso de medicamentos y plantas medicinales, con una mayor participación de profesionales de la salud.

Palabras clave: Ancianos, Automedicación, Profesionales de la salud.

\section{INTRODUÇÃO}

O uso inadequado de medicamentos constitui um importante problema de saúde pública que afeta negativamente a resposta aos tratamentos e aumenta os custos relacionados ao manejo de problemas relacionados às drogas (DRPs). O DRP refere-se a eventos de tratamento medicamentoso que podem interferir em seus resultados, sendo uma causa frequente de morbidades, hospitalizações e mortalidades, podendo estar associados a aspectos como necessidade de indicação, eficácia do tratamento, segurança, que inclui detecção e prevenção de reações adversas e medicamentos e adesão à terapia medicamentosa (RODRIGUES JPB, et al., 2019).

Observa-se um crescimento cada vez maior de idosos no mundo, associado principalmente a elevação da expectativa de vida da população. Somado a isso, os medicamentos passam a ser visto e utilizados mais amplamente pela maioria das pessoas e, como consequência disso, os efeitos deletérios pelo mau uso aumentaram, em que se estima cerca de 5,0 a 10,0\% das internações hospitalares ocorreram devido a DRPs, sendo que até $60,0 \%$ seriam evitáveis (ROZENFELD S, 2003; HOWARD RL, et al., 2007; SEKHAR MS, et al., 2011).

Portanto, no modelo assistencial vigente, o Enfermeiro vem desempenhando cada vez mais um importante papel na equipe de saúde, sendo um dos profissionais a ensinar os consumidores de saúde o uso adequado, os benefícios e os possíveis riscos dos medicamentos, assumindo a função de identificar as dificuldades e os fatores de riscos para que o paciente siga de forma eficaz o tratamento farmacológico prescrito pelo médico (COSTA ECVS, 2014), o que poderia contribuir para a redução nas internações relacionadas aos eventos da DRP, levando, especialmente, a otimização do uso de recursos financeiros associados à prestação de cuidados de saúde aos pacientes idosos (RODRIGUES JPB, et al., 2019).

De fato, sabe-se que os pacientes idosos são mais afetados pelos eventos da DRP, principalmente por serem polimedicados, pois o envelhecimento acomete órgãos e tecidos, elevando a prevalência de doenças crônicas não transmissíveis, tais como hipertensão arterial sistêmica, doenças osteoarticulares, diabetes mellitus e outras, o que aumenta o uso medicamentos como forma paliativa para o alívio destas enfermidades. Com isso, os medicamentos representam um dos itens mais importante à saúde do idoso e necessitam de atenção especial (FILHO AIL, et al., 2002; FERREIRA WA, et al., 2005).

O idoso é particularmente propenso a apresentar reações adversas ao tomar qualquer medicamento, podendo ser leves ou intensos. Esses efeitos podem aparecer após a primeira dose, várias doses, ou após a medicação ser suspensa, e até mesmo quando a medicação foi dada com sucesso por longo período (ROACH SS, 2009). Portanto, precisam de cuidado especial ao fazerem uso de medicamentos, visto que, sua idade avançada lhe submete a mudanças em seu metabolismo (ARAÚJO AL, et al., 2015). 
Contudo, há uma divergência grande entre a quantidade de idosos que se automedicam, variando de 17,7 a $31,2 \%$ nos países desenvolvidos e de 8,9 a $80,5 \%$ naqueles em desenvolvimento, o que pode ser explicado pela diferença de métodos adotados para avaliar a automedicação (MARTINS AP, et al., 2002). Além disso, poucos são os estudos (CARRASCO-GARRIDO P, et al., 2008; DE BOLLE L, et al., 2008; OLIVEIRA MA, et al., 2012) que avaliam a automedicação nesta faixa etária da população, demonstrando a necessidade de mais estudos.

Sabe-se que a prática da automedicação na sociedade atual deve ser vista como problema preocupante, pois cada dia cresce o número de pessoas que procuram a cura para os sintomas que lhes afligem a saúde em medicamentos indicados por familiares, amigos ou até mesmo por profissionais não comprometidos com a saúde da população, sendo que a maioria destas pessoas desconhecem as reações adversas graves geradas pelo uso indiscriminado de medicamentos (KATZUNG BG, 2014).

No Brasil, o acesso à assistência médica pública é difícil e existe uma grande parcela da sociedade em condições socioeconômicas precárias, a prática da automedicação torna-se bastante comum, justificada pela baixa situação financeira, escolaridade, escassez no acesso às informações a respeito dos medicamentos e, principalmente, ao fator cultural, o qual se baseia no uso de medicamentos sem receita médica (NASCIMENTO A, 2012).

Soma-se a isso, as pressões sociais em que os prescritores estão submetidos, a estrutura precária do sistema de saúde pública e o marketing farmacêutico (DALL'AGNOL RSA, 2004). Portanto, esses fatores citados tornam a automedicação um problema de saúde pública, na qual o mercado disponibiliza cada vez mais medicamentos que geram maior familiaridade com o usuário leigo através dos inúmeros tipos de propaganda que estão sujeitos (ZAMUNER CP, 2006).

Também a presença de grandes quantidades de medicamentos em residências se torna um risco à saúde, o que pode favorecer a prática da automedicação, possíveis trocas de medicações e intoxicações acidentais por ingestão, especialmente por crianças e animais (FILHO AIL, et al., 2002). Com isso, a farmácia caseira pode afetar a eficiência e a segurança no uso de medicamentos. Para isso, faz-se necessário analisar as causas da automedicação no paciente idoso, de modo a sensibilizar essa população dos perigos dessa prática, uma vez que pode desencadear complicações sérias para a saúde.

\section{MÉTODOS}

O tipo de estudo foi uma pesquisa de campo do tipo descritiva exploratória com abordagem quantitativa, a qual foi realizada na Unidade Municipal de Saúde (UMS) da Marambaia, localizada no município de Belém, estado do Pará. Possui serviços de urgência e emergência em nível ambulatorial, com atendimento de baixa e média complexidade e receber os pacientes por demanda espontânea e referenciada, oferecendo as seguintes áreas Clinica Geral, Pediatria, Ginecologia, Odontologia, Enfermagem e também conta com a operação da Assistência Médica Ambulatorial (AMA), da Estratégia de Saúde da Família (ESF) e do Programas de Saúde da Família (PSF). Apresenta domínio administrativo e gestão municipal.

O público alvo dessa pesquisa foi idoso matriculados na unidade, em que a população total era de 4.322 idosos, porém a amostra foi de 200 idosos, mas somente 112 responderam todo o questionário. Os critérios de inclusão foram idosos de ambos os sexos, a partir de 60 anos que aceitaram participar de livre e espontânea vontade da pesquisa e estavam matriculados na UMS. Foram excluídos idosos que procurarem a UMS por urgência/emergência, que não residem nas proximidades da unidade e os que deixarem o questionário incompleto ou sem responder.

Este estudo foi aprovado pelo comitê de ética da Universidade Paulista (UNIP) sob o número do CAAE (04620918.1.0000.5512) e parecer (3.180.115/2019), em consonância com a Resolução 466/12 do CONEP. Os idosos incluídos na pesquisa, após a assinatura do Termo de Consentimento Livre e Esclarecido (TCLE), foram identificados por pseudônimos por questões de privacidade (código alfa numéricos: A1, A2, B1, B2). Foram aplicados questionários aos idosos com aproximadamente 17 categorias. A aplicação dos questionários aconteceu in locu, durante as consultas. 
Após a coleta dos dados, o armazenamento foi realizado em planilhas do Microsoft Excel, organizado em categorias e respostas para serem posteriormente avaliados pelo teste qui-quadrado $(x 2)$ de Pearson, utilizando o programa Biostat 5.3, com nível de significância aceito de $5 \%(p \leq 0,05)$.

\section{RESULTADOS}

Foram entrevistados 200 idosos, mas somente 112 responderam. A maioria dos participantes eram do sexo feminino $(62,5 \%)$, de 60 a 80 anos $(94,6 \%)$, com ensino fundamental $(61,6 \%)$, casados $(36,6 \%)$ e aposentados ou pensionistas $(71,4 \%)$; (Tabela 1$)$.

Tabela 1 - Perfil dos idosos que participaram do estudo $(n=200)$.

\begin{tabular}{ccc}
\hline Característica & $\mathbf{n}=\mathbf{1 1 2}(\mathbf{n})$ & Porcentagem (\%) \\
\hline \multirow{2}{*}{ Gênero } & Masculino (42) & 37,5 \\
& Feminino (70) & 62,5 \\
\hline \multirow{2}{*}{ Idade } & $60-80$ anos (106) & 94,6 \\
& Acima de 80 anos (6) & 5,4 \\
\hline \multirow{2}{*}{ Escolaridade } & Analfabetos (5) & 4,5 \\
& Fundamental (69) & 61,6 \\
& Médio (31) & 27,7 \\
& Superior (7) & 6,2 \\
\hline \multirow{2}{*}{ Estado Civil } & Solteiro (20) & 17,9 \\
& Casado (41) & 36,6 \\
& Divorciado (13) & 11,6 \\
& Viúvo (33) & 29,5 \\
& Outros (5) & 4,4 \\
\hline \multirow{2}{*}{ Situação Profissional } & Empregados (11) & 9,8 \\
& Desempregados (2) & 1,8 \\
\hline \multirow{2}{*}{ Convênio Médico } & Aposentados ou Pensionistas (80) & 71,4 \\
& Do lar (19) & 17 \\
\hline
\end{tabular}

Fonte: Mello AGNC, et al., 2020.

Observou-se que as mulheres fazem mais uso de medicação $(p=0,0287)$ e leem mais a bula $(p=0,0013)$ que os homens (Gráfico 1), bem como a leitura desta está atrelada ao elevado grau de escolaridade $(\mathrm{p}=0,0078)$ (Gráfico 2).

Entretanto, a automedicação diminui com o aumento da idade ( $p=0,0245)$; (Gráfico 3). Já o uso de plantas medicinais e fitoterápicos é realizado pelos idosos de 60 a 80 anos $(p=0,0208)$ e aqueles com baixa ou sem renda comprovada ( $p=0,0103)$; (Gráfico 4). E a baixa escolaridade leva ao desconhecimento do tempo de manifestação dos sintomas iniciais que induziram ao uso indiscriminados de medicamentos $(p=0,0067)$, bem como influencia na diminuição $(p=0,0022)$ da procura por atendimento médico ou profissional de saúde para o uso correto de medicações, o que leva ao aumento da aquisição de medicamentos, associado a facilidade de compra (Gráfico 5). 
Gráfico 1 - Participantes $(n=200)$ que utilizam outros medicamentos e realizam a leitura da bula.

A Utilizam outros medicamentos

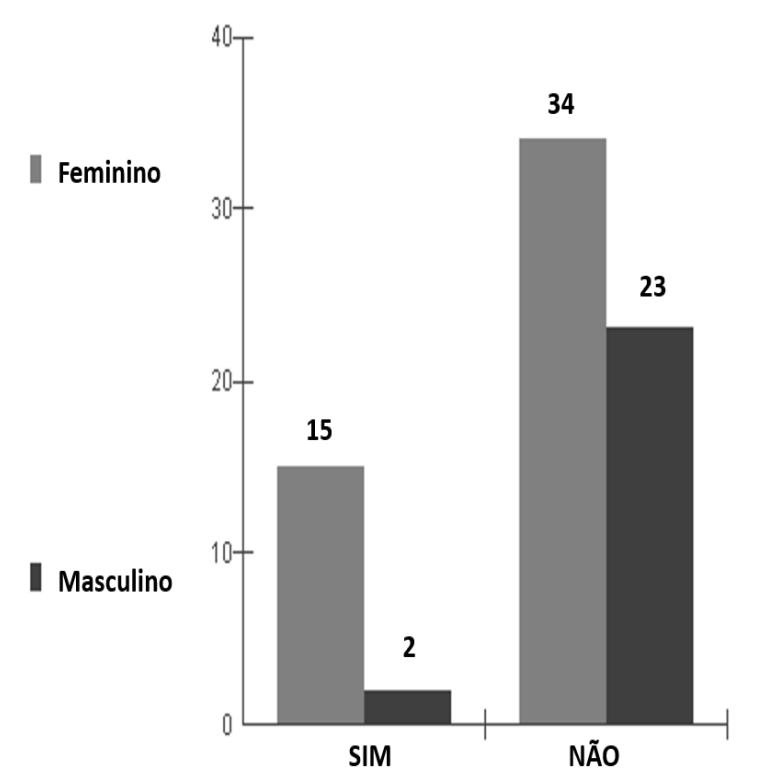

B Leitura da Bula de Medicamentos

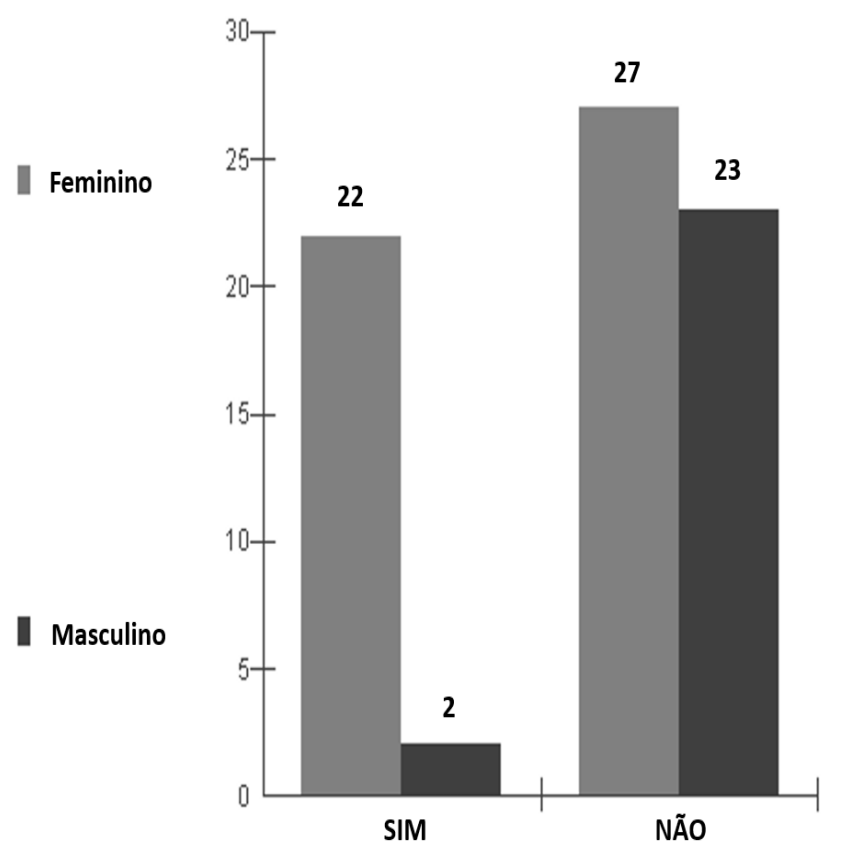

Legenda: A: O sexo feminino apresentou maior quantidade de participantes que fazem uso de outros medicamentos ( $p=0,0287)$. B: O sexo feminino lê a bula dos medicamentos com maior frequência $(p=0,0013)$. Fonte: Mello AGNC, et al., 2020.

Gráfico 2 - Correlação entre o grau de escolaridade e a realização da leitura da bula dos participantes $(n=200)$.

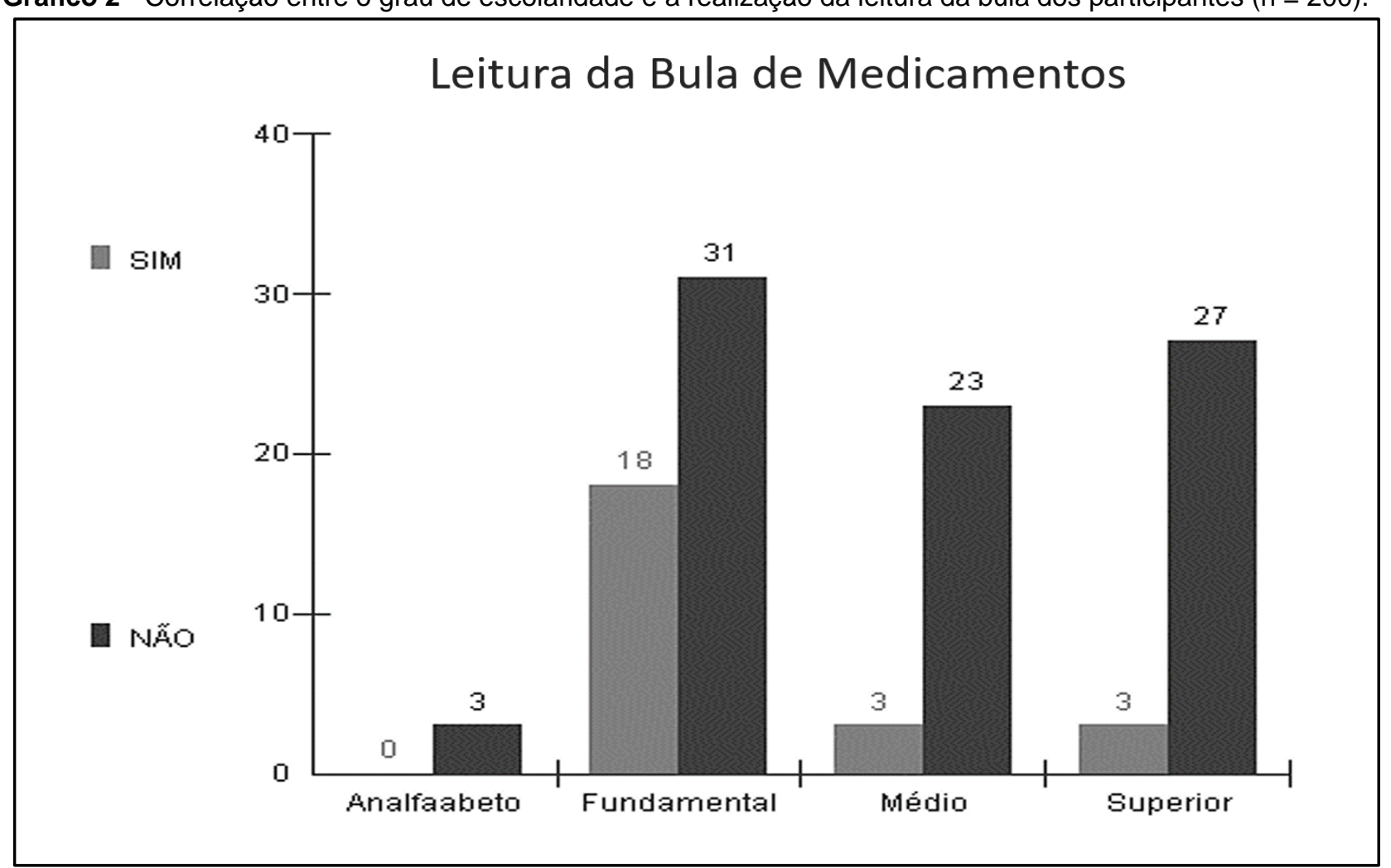

Fonte: Mello AGNC, et al., 2020. 
Gráfico 3 - A idade influência na automedicação dos participantes $(n=200)$.

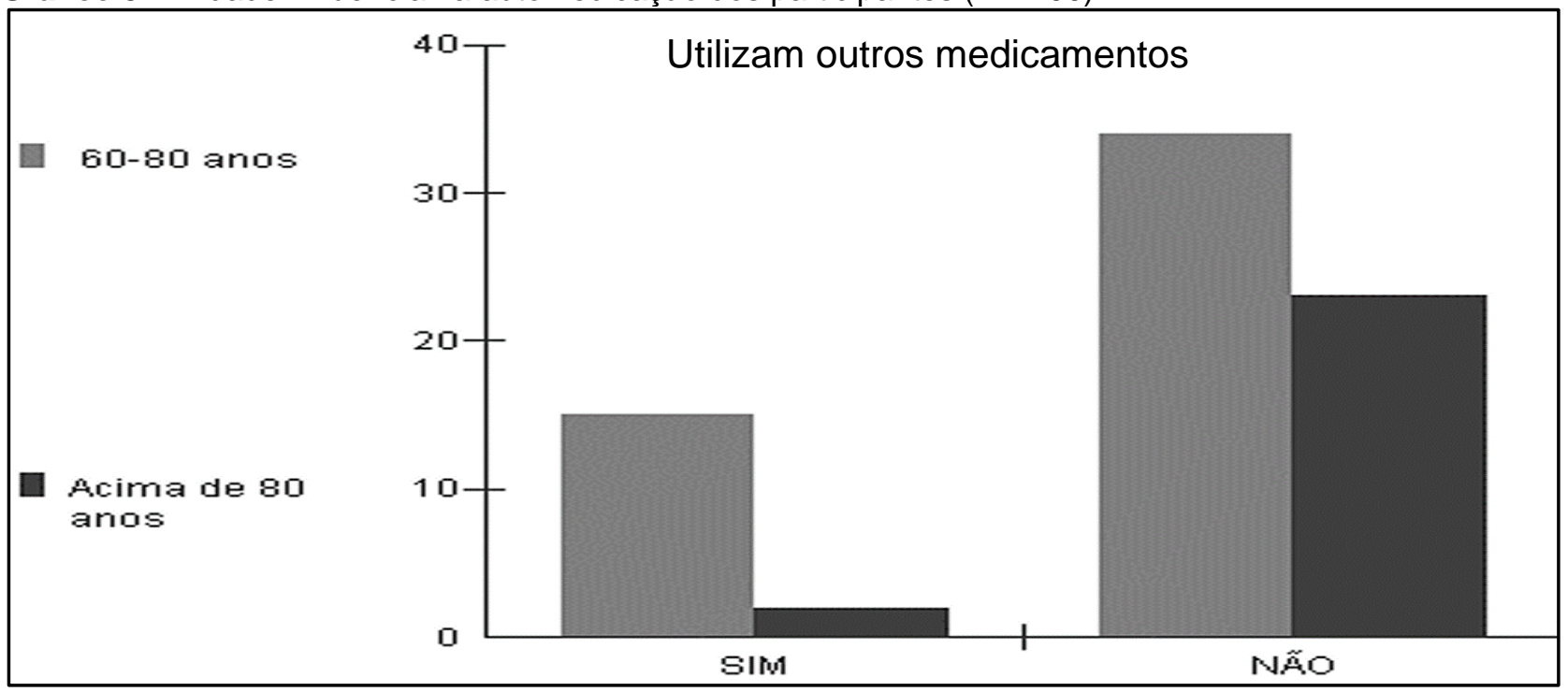

Fonte: Mello AGNC, et al., 2020.

Gráfico 4 - Idosos ( $n=200)$ utilizam mais plantas medicinais e fitoterápicos devido ao baixo poder aquisitivo.

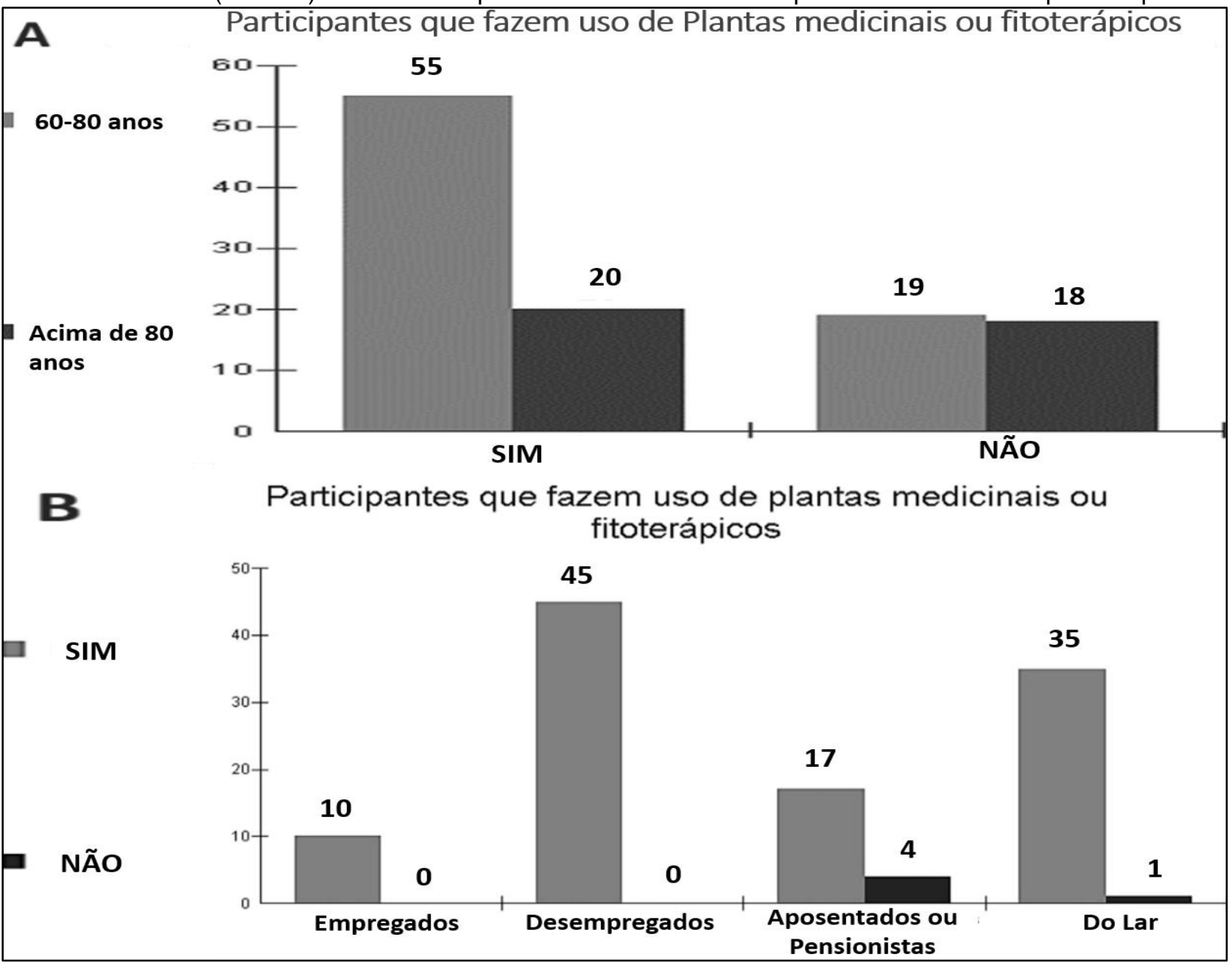

Legenda: A: Quanto menor a idade do idoso, maior é o uso de plantas medicinais e fitoterápicos ( $p=0,0208)$.B: Os desempregados e "do lar" são os idosos que mais utilizam plantas medicinais e fitoterápicos, demonstrando que o baixo valor desses tipos de terapias pode influenciar na aquisição por estes perfis de participantes $(p=0,0103)$.

Fonte: Mello AGNC, et al., 2020. 
Gráfico 5 - O baixo grau de escolaridade está atrelado ao desconhecimento do tempo de manifestação dos sintomas, bem como na diminuição da procura por atendimento médico dos participantes $(n=200)$. Belém, Pará, 2019.

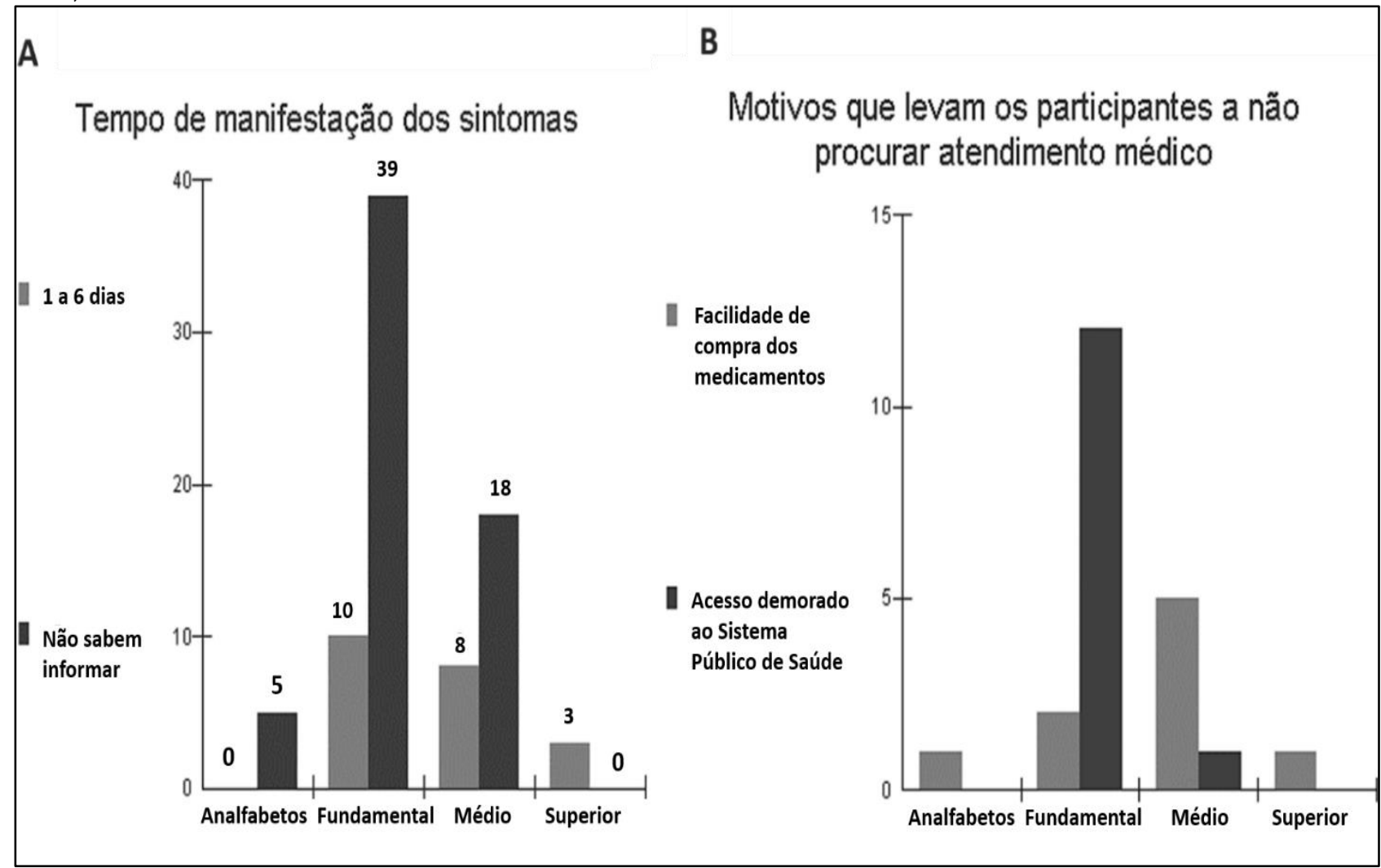

Legenda: A: Os idosos que possuíam ensino fundamental eram os que mais desconheciam o início das manifestações dos sintomas que levaram a automedicação $(p=0,0067)$. B: A facilidade da compra de medicamentos e a dificuldade do acesso ao sistema público de saúde levam os idosos com ensino fundamental a procurarem menos por serviço de profissionais de saúde para melhor orientação do uso correto de medicamentos $(p=0,0022)$.

Fonte: Mello AGNC, et al., 2020.

\section{DISCUSSÃO}

As mulheres representam cerca de $51,5 \%$ da população brasileira e a expectativa de vida dessas vem sendo apontada como maior em relação aos homens (IBGE, 2010), o que justifica a quantidade de $62,5 \%$ dos participantes serem do sexo feminino. Esses dados corroboram com outros estudos realizados que afirmam que atualmente tem ocorrido a feminização do envelhecimento, observado pelo elevado grau de viuvez entre as mulheres (43,6\% sobre 17,4\% dos homens) (POLARO SH, et al., 2013; ALMEIDA AV, et al., 2015). Porém, a maioria da população brasileira (51\%) possui apenas o ensino fundamental completo (IBGE, 2010), fato que corrobora com os resultados do estudo (Tabela 1).

De fato, os idosos tendem a apresentar baixa escolaridade, o que leva uma condição social desfavorável, podendo afetar na compreensão de seu tratamento, no seu autocuidado e em desvantagens socioeconômicas (SANTOS GS e CUNHA ICKO, 2017), as quais foram observadas em um estudo em Belém (PA), no qual $74,1 \%$ dos idosos da amostra tinham condições financeiras baixas, recebendo de 1 a 3 salários (PERES MAC, 2011).

Entretanto, as mulheres tendem a ter um maior grau de instrução que os homens (BRASIL, 2005). De fato, a educação tem sido um fator importante para o progresso profissional de muitas mulheres, com consequente ascensão feminina contínua, o que leva ao processo de inclusão educacional muito relevante porque tem reflexos na participação destas nos melhores postos de trabalho do país, sendo notórias as conquistas na busca por um melhor nível educacional, especialmente no que se refere ao nível superior (LEONE ET e PORTILHO L, 2018). 
Portanto, é esperado que a mulheres se informem mais que os homens, fato observado na maior participação delas durante a obtenção de informações sobre os medicamentos através da leitura da bula (Gráfico 1).

Uma bula contém várias informações técnicas de um determinado medicamento, como ação da droga, posologia, reações adversas, interações medicamentosas e contraindicações, sendo parte obrigatória da embalagem do produto.

A leitura é essencial na busca por mais informações que tragam mais segurança sobre o medicamento (RIGOTTO GC, et al., 2016). No entanto, os homens muitas vezes negligenciam sua importância, justificada pela falta de paciência de ler todas essas informações, conforme relato nos questionários respondidos.

De fato, os problemas com a leitura, compreensão do texto, tamanho da letra na maioria das vezes muito pequena, o exagero de informações em termos desconhecidos pelos pacientes, frases longas e complicadas e uso de normas em excesso apresenta-se como uma bula com problemas de interpretação.

Além disso, algumas bulas são consideradas difíceis de entender para os cidadãos com pouco estudo que sentem dificuldades no momento de uma leitura e de interpretar o que é lido ou por idosos. Mas a escolaridade em grau maior não significa que o paciente compreenderá o conteúdo das bulas, sendo informação segundo o Índice de Letramento Científico (RIGOTTO GC, et al., 2016).

Além disso, a redução da capacidade visual do idoso pode fazer com que este tenha dificuldades na leitura das informações contida no caixa dos medicamentos, bem como apresente problemas na leitura da bula, o que pode levar a uma interpretação errônea das informações, sendo potencial ao risco de intoxicação (SECOLI SR, et al. 2018). De fato, um estudo demonstrou que $12 \%$ dos idosos não leem a bula e outros $19 \%$ possuem dificuldade de entender a informação do rótulo, o que os levam a praticar a automedicação (NEAFSEY PJ, et al., 2007).

Observa-se que os idosos são provavelmente o grupo mais exposto à polifarmacoterapia na sociedade. A média de medicamentos utilizados por estes indivíduos é de dois a cinco medicamentos (BORTOLON PC, et al., 2008).

Alguns estudos têm demonstrado que as mulheres, em relação à idade, formam o grupo social que mais utiliza medicamentos, provavelmente por consequência de um pior estado funcional e de saúde auto referidos e evidenciado por maior número de sintomas de depressão e hospitalizações (OLIVEIRA MA, 2012; SANTOS GS e CUNHA ICKO, 2017). De fato, neste estudo foi observado que a mulheres são as que mais utilizam medicamentos que os homens (Gráfico 1).

Contudo, os idosos com idade de 60 a 80 anos eram os que mais se automedicavam, demonstrando que há uma redução do uso indiscriminado de medicamentos conforme o aumento da idade do idoso. Isso se contradiz com o estudo de Coelho Filho et al. (2004), o qual verificou maior chance de utilização de medicamentos exclusivamente prescritos entre idosos que referiram visita a serviços de saúde, presença de doença crônica, melhor nível socioeconômico e idade acima de 75 anos. Entretanto, nível econômico da maioria dos participantes deste estudo é baixo, o que pode ser explicado pela diminuição da aquisição de medicamentos.

Com isso, também observou que os idosos tendem a se automedicar mais com o uso de plantas medicinais e fitoterápicos, justificado pelo baixo poder aquisitivo da maioria e pelo baixo custo que essas finalidades terapêuticas possuem. Assim, enfatizam que a utilização destes esteja associada a fatores culturais, baixo custo, fácil aquisição e também não requerer receita médica (PETRY K e ROMAN JÚNIOR WA, 2012).

Além disso, as plantas medicinais são fáceis de manipular, geralmente as instruções de uso já vem na própria embalagem do produto, na maioria das vezes, as ervas já se encontram desidratadas, prontas para o uso em chás e infusões, o que garante mais praticidade ao seu uso (CESAR CLG, et al., 2005). Outra vantagem ressaltada pelos participantes da pesquisa é que as plantas raramente produzem efeitos colaterais significativos quando usadas corretamente, ao contrário dos fármacos, que muitas vezes trazem riscos. 
De fato, alguns estudos já observaram o uso de plantas medicinais mais frequente na população mais idosa, como por Araújo PL, et al. (2012), o qual notou 64,4\%, dos 135 idosos entrevistados no sul de Santa Catarina, utilizavam plantas medicinais para o tratamento de seus males; também Veiga VFJ (2008) observou que, do total de entrevistados, $65,6 \%$ realizavam a prática da automedicação por plantas na região CentroNorte do estado do Rio de Janeiro. E Melo WS, et al. (2019) notaram a utilização de chás ou outros, como forma de tratamento $(34,6 \%)$, sendo o problema que mais levou a utilizar o chá foi a insônia $(23,1 \%)$.

Assim como todo tratamento, as plantas medicinais e fitoterápicos também tem suas desvantagens, a maioria dos participantes acreditavam que, por ser um método natural, não existia riscos ou contraindicações. Porém, a utilização de plantas medicinais pode desencadear efeitos adversos, sendo estes decorrentes de inúmeros fatores, como, por exemplo, falta de conhecimento sobre as condições de cultivo, incorreta identificação farmacobotânica, informações insuficientes sobre reações adversas, esquema posológico, tempo de uso da terapia, e em especial, as interações medicamentosas (BARATA RB, 2008).

Por isso, é preciso lembrar que, apesar de serem produtos naturais, as plantas não são de todo inofensivas e podem causar alguns efeitos adversos devido às substâncias em sua composição, sendo importante utilizar um método de acompanhamento do uso de fitoterapia pelos profissionais de saúde.

Assim, o enfermeiro, por integrar uma equipe multiprofissional de saúde, pode promover o uso racional de plantas medicinais, através da educação permanente com o corpo técnico na discussão de casos clínicos sobre possíveis interações entre este tipo de terapêutica com os medicamentos tradicionais, bem como tentar educar a população no intuito de obter informações seguras e corretas sobre o uso de plantas e/ou fitoterápicos concomitantemente com os medicamentos.

Desse modo, estratégias poderão ser propostas e aplicadas após o conhecimento da necessidade e realidade daquela população em que estes profissionais atuam, fazendo da enfermagem uma das áreas mais atuantes, talvez pelo fato de realizar um atendimento multidimensional, humanizado e integral, e por atuar como promotora da saúde (BRASIL, 2009; CARVALHO TB, et al., 2015; SZERWIESKI LLD, et al., 2017).

Contudo, para se ter acesso ao serviço de saúde médico-ambulatorial é necessário conhecimento através de uma interação de fatores, como a necessidade e a percepção desta sob a ótica do usuário, e a oferta disponível de serviços.

Diversas condições associam-se a essa oferta, de maneira que a acessibilidade geográfica e os fatores socioculturais e econômicos, por exemplo, têm papel fundamental na demanda direcionada aos serviços de saúde (ECLIPSE COLLABORATIVE MEMBERS, et al., 2010).

Desse modo, observou-se que a baixa escolaridade foi um fator relatado pelos participantes que dificulta o acesso aos serviços públicos de saúde. De fato, Dilélio AS, et al. (2014) notou que a utilização de atendimento médico-ambulatorial foi significativamente maior entre os adultos das classes econômicas A/B, com 12 anos ou mais de escolaridade, com renda superior a um salário mínimo, residentes na Região Sul.

Também o baixo nível escolar dos participantes afetou a determinação do tempo inicial da manifestação dos sintomas que levaram a automedicação. Isso pode estar relacionado ao fato que pessoas que permanecem mais tempo no sistema educacional parecem ser mais capazes de compensar os eventuais efeitos da demência em seus cérebros do que as que estudaram menos (ECLIPSE COLLABORATIVE MEMBERS, et al., 2010). Assim, o elevado nível escolar seria uma medida de proteção da memória ao cérebro.

Portanto, acredita-se que seja necessária uma adaptação da sociedade para a correta aquisição das informações sobre os medicamentos de venda-livre e o uso de plantas medicinais e fitoterápicos, acerca do mecanismo de ação, reações adversas e contraindicações, que levem ao uso correto e consciente, impedindo o estímulo ao consumo desenfreado ou ao mito de cura milagrosa.

Ao mesmo tempo, incentiva-se a procura do profissional médico ou profissionais da saúde capacitados para evitar interações medicamentosas, as quais foram os problemas mais ressaltados pelos idosos que participaram do estudo. 


\section{CONCLUSÃO}

A partir dos resultados, conclui-se que os idosos procuram mais as plantas medicinais e os fitoterápicos para realizar a automedicação, justificada pelo baixo poder aquisitivo, dificuldade de acessibilidade aos serviços de saúde, bem como a baixa escolaridade. Além disso, muitos desconhecem o tempo inicial dos sintomas que levaram a automedicação, fato que pode ser justificado pela idade associado ao baixo nível escolar. Também se observou que as mulheres predominaram no estudo e fizeram mais uso de medicamentos, o que provavelmente esteja atrelado ao pior estado funcional e de saúde auto referidos e evidenciado por estas. Contudo, há necessidade de mais estudos que investiguem a automedicação no idoso, faixa etária que tem aumentado cada vez mais no país, necessitando mais políticas públicas de orientação à saúde.

\section{AGRADECIMENTOS}

Os autores deste trabalho agradecem à Secretária Municipal de Saúde do Belém, Pará, a Faculdade Pan Amazônica (FAPAN), pelo apoio durante a pesquisa, e aos idosos voluntários que participaram de maneira graciosa.

\section{REFERÊNCIAS}

1. ALMEIDA AV, et al. A Feminização da Velhice: em foco as características socioeconômicas, pessoais e familiares das idosas e o risco social. Textos Context (Porto Alegre) [Internet]. 2015; 14(1):115-31.

2. ARAÚJO AL, et al. Estudos brasileiros sobre automedicação: uma análise da literatura. Rev. Bras. Farm. 2015; 96(2): $1178-1201$.

3. BARATA RB. Acesso e uso de serviços de saúde: considerações sobre os resultados da Pesquisa de Condições de Vida 2006. São Paulo Perspect. 2008;22(2):19-29.

4. BORTOLON PC, et al. Análise do perfil de automedicação em mulheres idosas brasileiras. Ciênc. saúde coletiva. 2008; 13(4):1219-1226.

5. BRASIL. Ministério do Trabalho. Subsecretária de Recursos Humanos. Idosos brasileiros - indicadores de condições de vida e de acompanhamento de políticas. Brasília, 2005 [acesso 15 out 2018]. Disponível em: http://www.ipea.gov.br/portal/index.php?option=com_content\&view=article\&id=5476

6. BRASIL. Ministério da Saúde. Programa Nacional de Plantas Medicinais e Fitoterápicos [Internet]. Brasília: Ministério da Saúde, 2009 [acesso 16 abr 2017].

Disponível em: http://bvsms.saude.gov.br/bvs/publicacoes/programa_nacional_plantas_medicinais_fitoterapicos.pdf

7. CARRASCO-GARRIDO P, et al. Predictive factors of self-medicated drug use among the Spanish adult population. Pharmacoepidemiol Drug Saf 2008; 17(2): 193-9.

8. CARVALHO TB, et al. Papel dos Idosos no Contexto do Uso de Plantas Medicinais: Contribuições à Medicina Tradicional. Ensaios e Ciência: Ciências Biológicas, Agrárias e daSaúde [Internet]. 2015;19(1):38-41.

9. CESAR CLG, GOLDBAUM M. Usos de serviços de saúde. In: Cesar CLG, Carandina L, Alves MCGP, Azevedo MB, Goldbaum M. Saúde e condição de vida em São Paulo: inquérito multicêntrico de saúde no Estado de São Paulo. São Paulo: Faculdade de Saúde Pública da USP; 2005. p. 185-98.

10. COELHO FILHO JM, et al. Perfil de utilização de medicamentos por idosos em área urbana do Nordeste do Brasil. Rev Saúde Pública. 2004; 38:557-64.

11. COSTA ECVS. Toxicomania na sociedade contemporânea: Desafio para a prática profissional do Enfermeiro. Monografia (Curso de Especialização em Linhas de Cuidado em Enfermagem). Universidade Federal de Santa Catarina, Florianópolis, 2014, 32 p.

12. DALL'AGNOL RSA. Identificação e quantificação dos problemas relacionados com medicamentos em pacientes que buscam atendimento no serviço de emergência do HCPA. Dissertação (Mestrado em Ciências Farmacêuticas). Universidade Federal do Rio Grande do Sul, Porto Alegre, 2004, $112 \mathrm{p}$.

13. DE BOLLE L, et al. Home medication cabinets and self-medication: a source of potential health threats? Ann Pharmacother 2008; 42(4): 572-9.

14. DILÉLIO AS, et al. Padrões de utilização de atendimento médico ambulatorial no Brasil entre usuários do Sistema Único de Saúde, da saúde suplementar e de serviços privados. Cad. Saúde Pública. 2014; 30(12):2594-2606.

15. ECLIPSE COLLABORATIVE MEMBERS, et al. Education, the brain and dementia: neuroprotection or compensation? Brain 2010; 133: 2210-2216C.

16. FERREIRA WA, et al. Avaliação de Farmácia Caseira no Município de Divinópolis (MG) por Estudantes do Curso de Farmácia da Unifenas. Infarma. 2005; 17(7/9): 84-86.

17. FILHO AlL, et al. Prevalência e fatores associados à automedicação: resultados do Bambuí. Rev Saúde Pública. 2002; 36(1):55-62.

18. HOWARD RL, et al. Which drugs cause prevent- able admissions to hospital? A systematic review. $\mathrm{Br} J$ ClinPharmacol. 2007; 63(2):136-147.

19. IBGE. (Instituto Brasileiro de Geografia e Estatística). Censo demográfico 2010 [acesso 26 out 2018]. Disponível em: https://www.ibge.gov.br/estatisticas-novoportal/sociais/populacao/9662-censo-demografico2010.html?=\&t=downloads 
20. KATZUNG BG. Farmacologia Básica e Clínica. 12ª ed. Porto Alegre: AMGH, 2014.

21. LEONE ET, PORTILHO L. Inserção de mulheres e homens com nível superior de escolaridade no mercado de trabalho brasileiro. Temáticas 2018; 26 (52): 227-246.

22. MARTINS AP, et al. Self-medication in a Portuguese urban population: a prevalence study. Pharmacoepidemiol Drug Saf 2002; $11(5):$ 409-14.

23. MELO WS, et al. Prevalência de automedicação entre idosos acolhidos em um centro-dia. Revista Enf. Atual in Derme. 2019; 88 (26): 88-26.

24. NASCIMENTO A. Propaganda de medicamentos: como conciliar uso racional e a permanente necessidade de expandir mercados? Trab. educ. saúde. 2012; 5 (2):189- 215, 2012.

25. NEAFSEY PJ, et al. Self-medication practices of Spanish-speaking older adults in Hartford, Connecticut. Hisp Health Care Int 2007; 5(4): 169-79.

26. OLIVEIRA MA, et al. Automedicação em idosos residentes em Campinas, São Paulo, Brasil: prevalência e fatores associados. Cad Saúde Públ 2012; 28(2): 335-45.

27. PETRY K, ROMAN JÚNIOR WA. Viabilidade de implantação de fitoterápicos e plantas medicinais no Sistema Único de Saúde (SUS) do município de Três Passos/RS. Rev Bras Farm. 2012;93(1):60-7.

28. PERES MAC. Velhice e analfabetismo, uma relação paradoxal: a exclusão educacional em contextos rurais da região Nordeste. Soc Estado. 2011; 26(3):631-62.

29. POLARO SHI, et al. Condições de vida e saúde de idosos residentes em áreas ribeirinhas. Rev enferm UFPE on line., 2013; 7(9):5510-5517.

30. RIGOTTO GC, et al. A bula de medicamentos: a importância da leitura das bulas. Revista Científica da FAEMA. 2016; 7(1): 16-26.

31. ROACH SS. Introdução à Enfermagem Gerontológica. Rio de Janeiro: Guanabara Koogan, 2009; 351p.

32. RODRIGUES JPB, et al. Analysis of clinical pharmacist interventions in the neurology unit of a Brazilian tertiary teaching hospital. PloS ONE. 2019; 14(1): e0210779.

33. ROZENFELD S. Prevalência, fatores associados e mau uso de medicamentos entre os idosos: uma revisão. Cad. Saúde Públ 2003; 19(3): 717-24.

34. SANTOS GS e CUNHA ICKO. Fatores associados ao consumo de medicamentos entre idosos de uma unidade básica de saúde. REFACS [Internet]. 2017; 5(2):191-199.

35. SECOLI SR, et al. Tendência da prática de automedicação entre idosos brasileiros entre 2006 e 2010: Estudo SABE. Rev Bras Epidemiol 2018; 21(suppl 2): E180007.supl.2

36. SEKHAR MS, et al. Study on drug related admissions in a tertiary care hospital in South India. Saudi Pharm J. 2011;19(4):273-278.

37. SZERWIESKI LLD, et al. Uso de plantas medicinais por idosos da atenção primária. Rev. Eletr. Enf. [Internet]. 2017;19:a04.

38. VEIGA VFJ. Estudo do consumo de plantas medicinais na Região Centro-Norte do Estado do Rio de Janeiro: aceitação pelos profissionais de saúde e modo de uso pela população. Rev Bras Farmacogn [Internet]. 2008 Apr/June.

39. ZAMUNER CP. Prefeitura do Município de Tietê Secretaria Municipal de Saúde Vigilância Sanitária e Epidemiológica. Cuidado com os medicamentos. Jun. 2006 [acesso 05 out 2018]. Disponível em: http://www.tiete.sp.gov.br/ 